\title{
Benign testicular cavernous hemangioma presenting with acute onset: A case report
}

\author{
FUBIAO LI ${ }^{1}$, SHUMEI HAN ${ }^{2}$, LINGYUN LIU $^{1}$, SHENGQI XU ${ }^{1}$, DONGYANG CAI ${ }^{1}$, \\ ZUOWENG LIANG ${ }^{1}$, HONGLIANG WANG ${ }^{1}$ and KAIMIN GUO ${ }^{1}$
}

Departments of ${ }^{1}$ Andrology and ${ }^{2}$ Medical Affairs, The First Hospital of Jilin University, Changchun, Jilin 130021, P.R. China

Received July 12, 2019; Accepted March 31, 2020

DOI: $10.3892 / \mathrm{mco} .2020 .2033$

\begin{abstract}
Cavernous hemangioma is a congenital, benign vascular tumor that occurs in the deep dermis and subcutaneous tissue. Testicular cavernous hemangioma is extremely rare, mostly occurring during childhood or adolescence. Testicular cavernous hemangioma is a benign tumor that appears as a slowly growing painless mass. In rare cases, it may be associated with acute testicular infarction or torsion with acute onset. We herein report the case of a patient with an atypical presentation of testicular cavernous hemangioma, characterized by acute painful testicular enlargement triggered by minor injury. The patient underwent right radical orchiectomy, and histopathological examination confirmed the diagnosis of testicular cavernous hemangioma. Although this is a rare tumor, it should be considered in the differential diagnosis of testicular tumors.
\end{abstract}

\section{Introduction}

Vascular neoplasms of the testis and its adnexa are uncommon. Testicular cavernous hemangioma is a benign vascular neoplasm that may arise either within the testicular parenchyma (intratesticular) or from adnexal structures of the testis (extratesticular) $(1,2)$. In addition, testicular cavernous hemangiomas originating from the spermatic cord (3) and scrotum (4) have been reported. Overall, cavernous hemangiomas developing in the external genitalia are an infrequent occurrence, and cavernous hemangioma of the testis is extremely rare (5). This type of tumor may be encountered at any age, from fetuses of 17 weeks to patients aged 77 years (6), and its progression is slow (7). The aim of the present study was to report a rare case

Correspondence to: Dr Kaimin Guo, Department of Andrology, The First Hospital of Jilin University, 71 Xinmin Street, Changchun, Jilin 130021, P.R. China

E-mail: gkm119254097@126.com

Key words: testicular cavernous hemangioma, testicular benign tumor, acute onset of a testicular cavernous hemangioma in an adult manifesting with an acute onset.

\section{Case report}

A 23-year-old male patient presented with a tender right scrotal swelling for 7 days in June 2018. The patient had been seen by local physician and treated for epididymitis with antibiotics and scrotal support. However, the testicular pain persisted and the patient was referred to the andrology clinic of the First Hospital of Jilin University. The patient's medical history was unremarkable, and he had no previous history of trauma or infection. However, he reported an accidental blunt trauma to the scrotum 2 weeks earlier, with transient scrotal pain. On physical examination, a solid, tender, edematous mass was identified, measuring 20x20 mm, without transillumination. The tumor presented as a soft, spongy, non-pulsatile, painless, irregularly lobulated mass, not involving the ipsilateral epididymis or spermatic cord.

With clinical suspicion of testicular torsion, the patient was subjected to ultrasound examination, which demonstrated a hypoechoic lesion in the upper pole of the right testicle, with poorly demarcated margins and abundant blood flow (Fig. 1). No calcifications, fibrosis or necrotic foci were found in the area of the lesion. There was no extension to the perineum or groin, and no regional lymphadenopathy. Computed tomography examination clearly demonstrated a solitary tumor of the right testis (Fig. 2). The laboratory examinations, including relevant tumor markers, particularly $\alpha$-fetoprotein $(2.31 \mathrm{ng} / \mathrm{ml}$; normal range, $<20 \mathrm{ng} / \mathrm{ml}$ ), $\beta$-human chorionic gonadotropin $(0.11 \mathrm{ng} / \mathrm{ml}$; normal range, $<4 \mathrm{ng} / \mathrm{ml})$ and serum lactate dehydrogenase (171 U/1; normal range, 120-150 U/l) were normal.

The patient was diagnosed with a testicular tumor and a right inguinal incision was performed to access the spermatic cord and separate the right testis, together with the tunica vaginalis, from the scrotum. Intraoperatively, a solid, richly vascular tumor, sized $30 \times 20 \times 20 \mathrm{~mm}$, was identified on the upper pole of right testis (Fig. 3). The histopathological examination confirmed that the tumor was mainly composed of vascular lumina and thromboses, consistent with the diagnosis of testicular cavernous hemangioma (Fig. 4). The patient was discharged 1 week later, and remained free of complications and recurrence at the 1-year flow-up. 


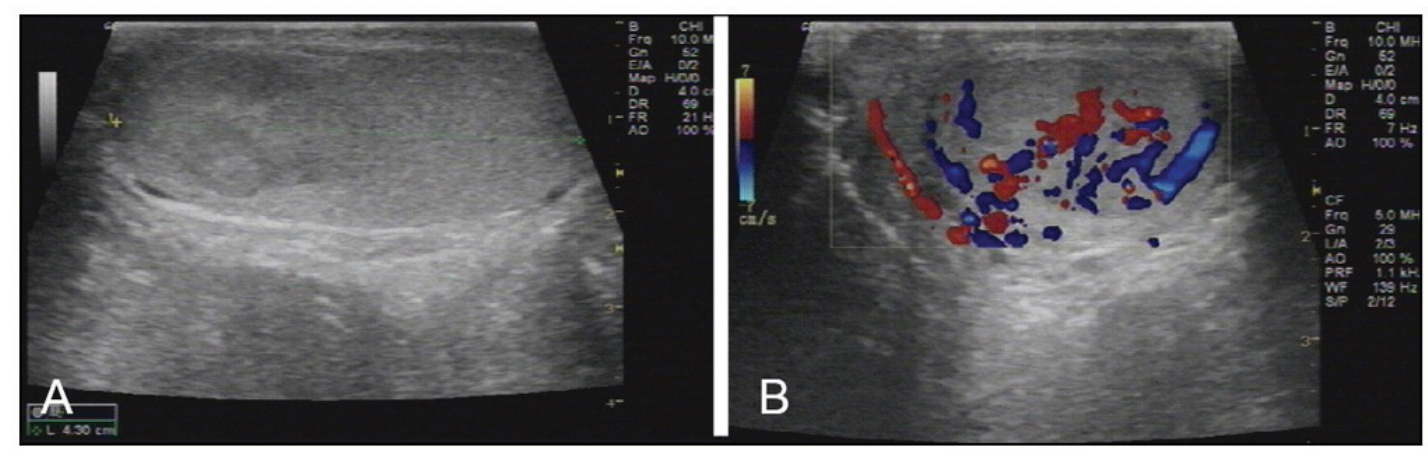

Figure 1. Scrotal ultrasound scan imaging. (A) A poorly-demarcated, hypoechoic mass without calcification was detected in the right testicle. (B) High blood flow was observed on color Doppler ultrasonography.
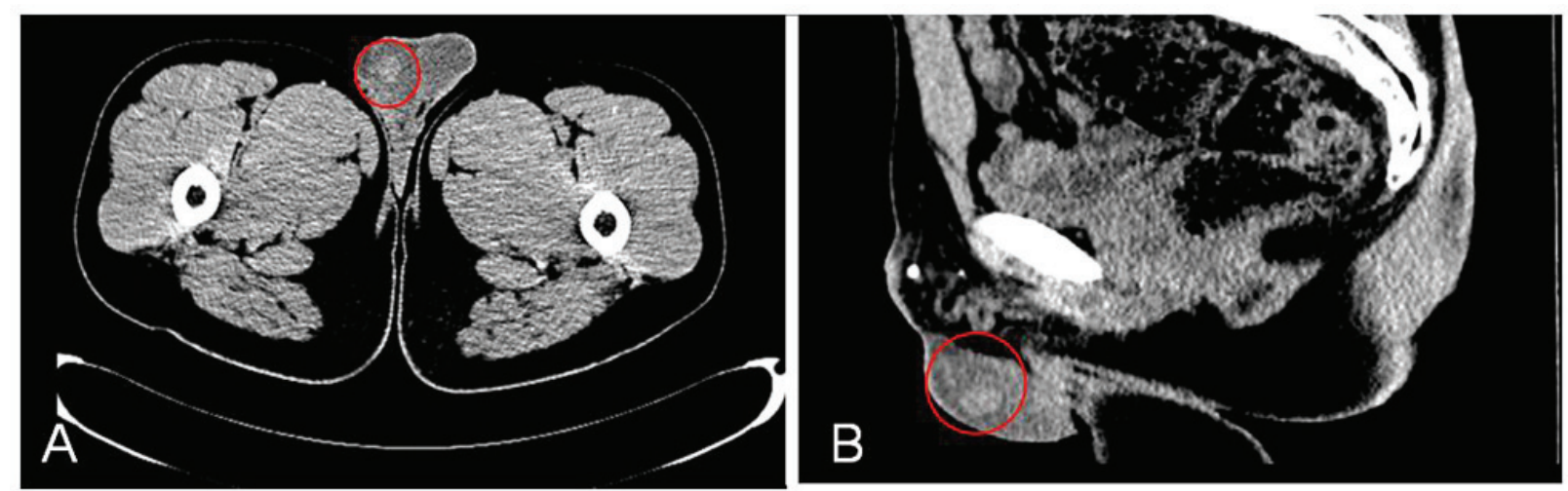

Figure 2. On computed tomography scan examination, a solid mass was (red circle) identified in the parenchyma of the right testis. (A) Transverse plane. (B) Sagittal plane.

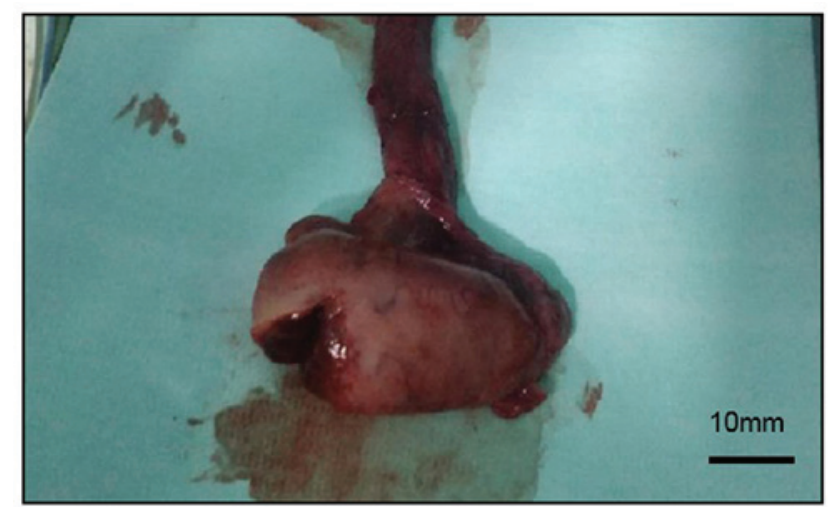

Figure 3. Macroscopic appearance of the testis after right radical orchiectomy. The involved testis was filled with hemorrhagic vascular tissue.

\section{Discussion}

Hemangioma is a type of the benign vascular tumor that may develop in any part of the body. In the pediatric population, hemangiomas located in the head and neck account for $60 \%$, with $25 \%$ found in the truck and $15 \%$ in the extremities, whereas only $2 \%$ of hemangiomas develop in the genitalia $(4,8)$. After Kleiman first reported a case of testicular hemangioma in 1944 (9), 50 cases of hemangiomas of the testis have been reported. Four histological types of hemangioma of the testis have been reported to date: Cavernous hemangiomas, capillary hemangiomas, histiocytoid and papillary endothelial hyperplasia. These occur in diverse age groups, but are most commonly encountered in children and adolescents. The youngest reported patient was a 1-month-old infant (10) and the oldest case was a 77 year-old man (7). Cavernous hemangiomas of the testis primarily present during childhood. Their clinical presentation varies from a painless testicular enlargement to painful testicular infarction $(8,11)$. Thus, the diagnosis is usually established only following inguinal orchiectomy. We herein describe the case of an adult patient presenting with acute onset of testicular enlargement and pain, which may be due to intratesticular bleeding caused by blunt testicular injury.

The differential diagnosis must include germ cell tumors (e.g., seminoma and teratoma), adenomatoid tumor and sex-cord stromal tumors, such as Sertoli cell tumor. Sonography is the primary modality used for imaging scrotal lesions. On sonographic examination, these tumors may present as hypoechoic, hyperechoic, or mixed echogenic masses, which may be either well or poorly defined. Color Doppler patterns may vary among different types of hemangiomas (capillary, cavernous or venous), as some hemangiomas display slower flow or a lesser degree of vascular pooling. Extensive hypervascularity and areas of low-resistance velocity on spectral Doppler imaging are considered to be suggestive of hemangioma (12). Furthermore, a testicular mass with various-sized calcifications is a characteristic presentation of testicular hemangioma (3). The calcifications may be phleboliths that 

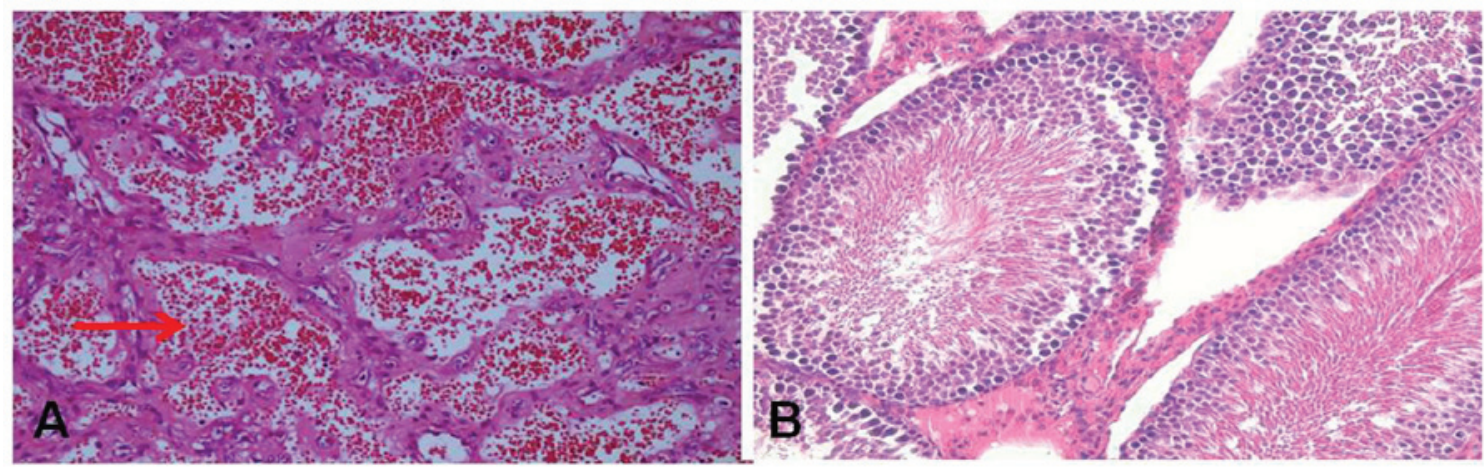

Figure 4. Hematoxylin and eosin staining revealed dilated thin-walled cavernous vascular spaces filled with red blood cells (arrow). (A) Histological examination revealed a benign tumor composed of proliferated vessels in the testicular parenchyma (magnification, x50); (B) normal testicular architecture is shown for comparison (magnification, $\mathrm{x} 100$ ).

frequently form in the dilated venous spaces of a cavernous hemangioma, or represent stromal calcifications (13). In the present case, there was no evidence of intratesticular calcification, and no thrombosis or calcification were detected on pathological examination. Thus, it may be difficult to distinguish this tumor from testicular carcinoma preoperatively using only these modalities. In addition, contrast-enhanced ultrasound (CEUS) has been suggested as an adjunctive tool for testicular imaging. CEUS is characterized by nodular enhancement around the arterial phase, progressive centripetal filling, and hyperechoic/isoechoic changes in the portal and delayed phases in CEUS images.

The available treatment options for testicular hemangioma include surgical excision, vessel ligation, intralesional sclerotherapy, radiation, intravascular steroid, laser fulguration, cryotherapy and orchiectomy $(14,15)$. The complications of testicular cavernous hemangioma include further enlargement, rupture, hemorrhage, infection, torsion, infarction and infertility. Intraoperative frozen section examination should be performed, as it is helpful for performing testicle-sparing surgery. All reported vascular testicular tumors have exhibited benign clinical behavior, without local recurrence or metastasis.

In conclusion, testicular cavernous hemangioma is an extremely rare disease, and its clinical presentation and findings on examination are similar with those of malignant testicular tumors, which may lead to diagnostic difficulties. Scrotal injury may trigger the onset of testicular swelling or pain. When dealing with a testicular mass in a patient without serum elevations of $\beta$-human chorionic gonadotropin or $\alpha$-fetoprotein, and ultrasound examination showing a mass with vascular proliferation, with or without calcifications, testicular cavernous hemangioma should be considered in the differential diagnosis.

\section{Acknowledgements}

Not applicable.

\section{Funding}

The present study was partially supported by the Jilin Finance Science Foundation (grant. no. JLSCZD2019-001)..

\section{Availability of data and materials}

All data generated or analyzed during the present study are included in the published article.

\section{Authors' contributions}

FL and SX performed the surgery. LL, ZL , DC and HW provided medical care for the patients and collected the data. SH collected the data and revised the manuscript. KG wrote this manuscript ansd analyzed all of the data. LL, ZL and DC provided medical care for the patients and collected the data. All the authors have read and approved the final version of the manuscript.

\section{Ethics approval and consent to participate}

The present study was approved by the Ethics Review Committee of The First Hospital of Jilin University.

\section{Patient consent for publication}

The patient provided written informed consent for the publication of the case details and associated images.

\section{Competing interests}

All the authors declare that they have no competing interests.

\section{References}

1. Erdag G, Kwon EO, Lizza EF and Shevchuk M: Cavernous hemangioma of tunica albuginea testis manifesting as testicular pain. Urology 68: 673.e13-5, 2006.

2. Chetty R, Bandid S and Freedman D: Cavernous haemangioma of the epididymis mimicking a testicular malignancy. Aust N Z J Surg 63: 235-237, 1993.

3. Jeon YS, Cho SG, Kim WH and Choi SJ: Cavernous haemangioma of the spermatic cord in a child. Pediatr Radiol 36: 1323-1325, 2006.

4. Ergün O, Ceylan BG, Armagan A, Kapucuoglu N, Ceyhan AM and Perk $\mathrm{H}$ : A giant scrotal cavernous hemangioma extending to the penis and perineum: A case report. Kaohsiung J Med Sci 25: 559-561, 2009.

5. Liu B, Chen J, Luo J, Zhou F, Wang C and Xie L: Cavernous hemangioma of the testis mimicking a testicular teratoma. Exp Ther Med 6: 91-92, 2013. 
6. Suriawinata A, Talerman A, Vapnek JM and Unger P: Hemangioma of the testis: Report of unusual occurrences of cavernous hemangioma in a fetus and capillary hemangioma in an older man. Ann Diagn Pathol 5: 80-83, 2001.

7. Frank RG, Lowry P and Ongcapin EH: Images in clinical urology. Venous cavernous hemangioma of the testis. Urology 52: 709-710, 1998.

8. Tepeneu NF, Krafka K, Meglic S, Rogatsch H and Fasching G: Testicular cavernous hemangioma associated with testicular torsion-case report and review of literature. Int J Surg Case Rep 49: 247-250, 2018.

9. Kleiman AH: Hemangioma of the testis. J Urol 51: 548-550, 1944.

10. Manoney MT: Cavernous hemangioma of the scrotal septum. J Pediatr 49: 744-745, 1956.

11. Fossum BD, Woods JC and Blight EM Jr: Cavernous hemangioma of testis causing acute testicular infarction. Urology 18: 277-278, 1981.

12. Ricci Z, Koenigsberg M and Whitney K: Sonography of an arteriovenous-type hemangioma of the testis. AJR Am J Roentgenol 174: 1581-1582, 2000.
13. Venkatanarasimha N, McCormick F and Freeman SJ: Cavernous hemangioma of the testis. J Ultrasound Med 29: 859-860, 2010.

14. Ray B and Clark SS: Hemangioma of scrotum. Urology 8: 502-505, 1976.

15. Naveed S, Quari H and Sharma H: Cavernous haemangioma of the testis mimicking testicular malignancy in an adolescent. Scott Med J 58: e5-e7, 2013.

(i) $(9)$ This work is licensed under a Creative Commons Attribution-NonCommercial-NoDerivatives 4.0 International (CC BY-NC-ND 4.0) License. 